Minireview

\title{
Plant-Derived Kavalactones and Their Bioactivities
}

\author{
Nenling Zhang, ${ }^{a, b}$ Linjing Wu, ${ }^{a, b}$ Xiang Liu, ${ }^{a, b}$ and Xiangchun Shen ${ }^{\star, a, b}$ \\ ${ }^{a}$ The High Educational Key Laboratory of Guizhou Province for Natural Medicinal Pharmacology and \\ Druggability, Guizhou Medical University, Guiyang, Guizhou 550025, China \\ ${ }^{b}$ The Union Key Laboratory of Guiyang City \& Guizhou Medical University, Guiyang, Guizhou 550025, China
}

Email: shenxiangchun@126.com (X. S.)

\begin{abstract}
Kavalactones, mainly found in Piper methysticum, are a class of lactone compounds usually with an arylethylene-pyrone skeleton. Till now, fifty-one kavalactones have been isolated from the genera Piper, Alpinia, Amomum, Polygala, Goniothalamus, and Dumortiera. The bioactivities of six kavalactones, kawain, 7,8-dihydrokawain, methysticin, dihydromethysticin, yangonin, and desmethoxyyagonin, have been studied extensively. This review aims to give an overview of kavalactones in the plants and their various bioactivities.
\end{abstract}

Keywords kavalactone, Piper, Alpinia, bioactivities

\section{Introduction}

Kavalactones, also called kavapyrones or styrylpyrones, are a class of lactones generally with an arylethylene-pyrone skeleton. ${ }^{[1]}$ They are mainly found in Piper methysticum G. Forst (kava: Piperaceae) and have once drawn researchers' attention due to their various bioactivities, especially for their anti-depress activities. Kava extract, containing large amount of kavalactones, had been commonly used as an anti-depressant until its ban in Europe in 2002, when several cases concerning alleged liver toxicity were reported. After that, culprits for causing hepatoxicity were investigated. ${ }^{[2-4]}$ Kavalactones, as the main constituents of kava extract, were also tested for hepatoxicity. ${ }^{[5,6]}$ However, there was lack of evidence that supported the alleged dangers related to kavalactones. With German court lifting ban on kava in $2014,{ }^{[7]}$ the research on kavalactones might flourish again. Herein, we will summarize the kavalactones from the plants and their bioactivities.

\section{Kavalactones isolated from plants}

\section{Kavalactones}

Eighteen kavalactones (1-18), which were identified from kava roots, have already been covered in the review by Bilia et al, ${ }^{[8]}$ namely 11-hydroxy-12-methoxydihydrokavain (1), 7,8dihydrokavain (2), dihydromethysticin (3), 5,6,7, 8-tetrahydroyangonin (4), 11,12-dimethoxy-5,6-dihydrokavain (5), 7,8-dihydro-5-hydroxykavain (6), kavain (7), methysticin (8), 5,6-dihydroyangonin (9), hydroxykavain (10), 5,6-dehydromethysticin (11), 7,8-dihydroyangonin (12), desmethoxyyangonin (13), yangonin (14), 11-methoxyangonin (15), 10-methoxyangonin (16), 11-hydroxyangonin (17), and 11-methoxy-12-hydroxydehydrokavain (18). Among these kavalactones, compounds 2, 3, 7, 8, 13,14 are the most abundant in the roots of $P$. methysticum. ${ }^{[9]}$ Compound 13 was also isolated from the genera Alpinia and Amomum ${ }^{[10-12]}$ Chromatographic separation of the methylene chloride extract of $P$. methysticum roots yielded a new kavalactone 11-methoxy-5,6-dihydroyangonin (19). ${ }^{[13]}$ Dihydro5,6-dehydrokavain (DDK, 20), a main constituent in the leaves of Alpinia zerumbet, was determined using GC-MS by Kuster et al. ${ }^{[14]}$ Six kavalactones, 4-methoxy-6-(11,12-methylenedioxydihydrostyryl)-2-pyrone (21), 4-methoxy-6-(11,12-methylenedioxy-14-methoxy-dihydrostyryl)-2-pyrone (22), 4-methoxy-6(11,12-methylenedioxy-10,14-dimethoxydihydrostyryl)-2-pyrone (23), 4-methoxy-6-(11,12-methylenedioxy-14-methoxystyryl)-2pyrone (24), 4-methoxy-6-(11,12-methylenedioxystyryl)-2pyrone (25), and 4-methoxy-6-(11,12-dimethylstyryl)-2-pyrone (26) were isolated from the EtOAc-soluble fraction of the aqueous EtOH (1:4) extract of Polygala sabulosa (Polygalaceae). ${ }^{[15]}$ Matsuda et al. isolated 7,8-epoxyyangonin ( 27 ) from the extract of the roots of $P$. methysticum. ${ }^{116]}(+)$-Goniothalamin (28) and goniodiol (29), with no substituent on the ring of pyrone, were found in Cryptocarya moschatam (Lauraceae $)^{[17]}$ and the genus Goniothalamus. ${ }^{[18]}(+)$-Altholactone (30), $(+)$-garvensintriol (31), (+)-etharvendiol (32), and (-)-etharvensin (33) were isolated from the stem bark of Goniothalamus arvensis, and (+)-altholactone was identified as a major compound. ${ }^{[19-21]}$ Alkofahi et al. isolated goniotriol (34) from Goniathalamus giganteus and settled its relative configuration by single X-ray diffraction. ${ }^{[22]}$ Three carboxylated-pyrone derivatives, Dumortins A-C (35-37), were isolated from Dumortiera hirusa. ${ }^{[23]}$

\section{Kavalactone dimers}

Diyangonins $A-C(38,39,41)$, yangonindimers $A-C$ (43-45), and three known anologues [(rel-trans-3-bis[6(4-methoxy-2-pyronyl)]-cis-2,trans-4-diphenyl cyclobutane (40) 6,6'-(3,4-diphenylcyclobutane-1,2-diyl)bis(4-methoxy-2H-pyran2-one) (42), aniba-dimer A (46)], were isolated from roots of $P$. methysticum. ${ }^{[2,25]}$ Kavalactone dimers $\left(r-8, c-7^{\prime}, t-7-8,8^{\prime}-11,12\right.$ dimethoxyphenyl-7, $7^{\prime}$-di-[6-(4-methoxy-2-pyronyl)]-cyclobutane, 47) and (rel-(6R,7S,8S,5'S)-4'-methoxy-8-(11,12-dimethoxyphenyl)-7-[6-(4-methoxy-2-pyronyl)]-6-(E)-styryl-1'-oxabicyclo[4 $, 2,0]$ octa- 4 -en-2'-one, 48) were isolated from the trunk wood of Aniba species. ${ }^{[26]}$

\section{Hybrids of kavalactone and other types}

Three hybrids, katsumadain A (49), katsumadain B (50), and katsumadain (51), which were fused by kavalactone and diarylheptanoid or terpene, have been isolated from the seeds of Alpinia katsumadai. ${ }^{[27-29]}$ 

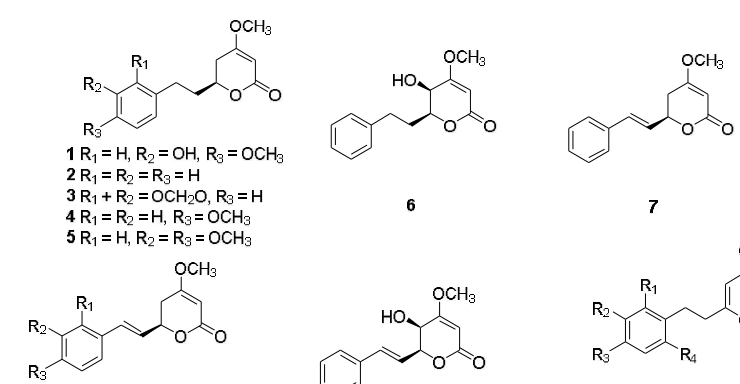

$8 \mathrm{R}_{1}+\mathrm{R}_{2}=\mathrm{OCH}_{2} \mathrm{O}, \mathrm{R}_{3}=\mathrm{H}$ $9 R_{1}=R_{2}=H_{1} R_{3}=O C_{3}$
$19 R_{1}=R_{2}=H, R_{2}=R_{3}=O_{3}$
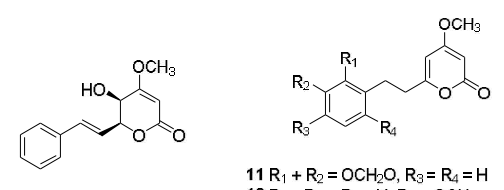

10

$11 \mathrm{R}_{1}+\mathrm{R}_{2}=\mathrm{OCH}_{2} \mathrm{O}, \mathrm{R}_{3}=\mathrm{R}_{4}=\mathrm{H}$ $12 R_{1}=R_{2}=R_{4}=H, R_{3}=O C H_{3}$ $20 R_{1}=R_{2}=R_{3}=R_{4}=H$
$21 R_{1}=R_{4}=H, R_{2}+R_{3}=O H_{2} O$ $21 \mathrm{R}_{1}=\mathrm{R}_{4}=\mathrm{H}_{1} \mathrm{R}_{2}+\mathrm{R}_{3}=\mathrm{OCH}_{2} \mathrm{O}$
$22 \mathrm{R}_{1}=\mathrm{OCH}_{3}, \mathrm{R}_{2}+\mathrm{R}_{3}=\mathrm{OCH} \mathrm{O}_{2} \mathrm{R}_{4}=\mathrm{H}$ $22 \mathrm{R}_{1}=\mathrm{OCH}_{3}, \mathrm{R}_{2}+\mathrm{R}_{3}=\mathrm{OCH}_{2} \mathrm{O}, \mathrm{R}_{4}=$
$23 \mathrm{R}_{1}=\mathrm{R}_{4}=\mathrm{OCH}_{3}, \mathrm{R}_{2}+\mathrm{R}_{3}=\mathrm{OCH} \mathrm{OH}_{2} \mathrm{O}$

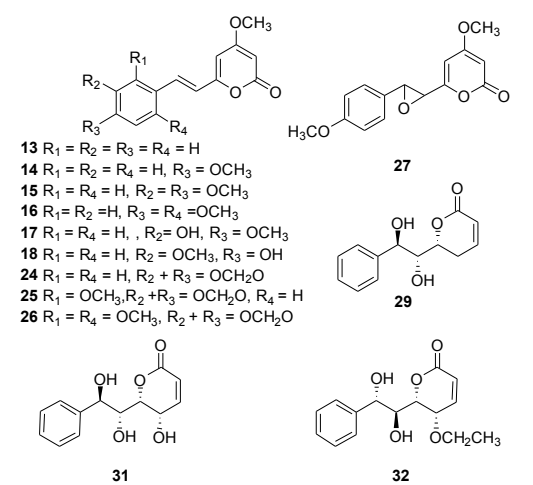

3

32

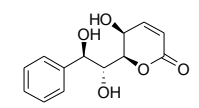

34
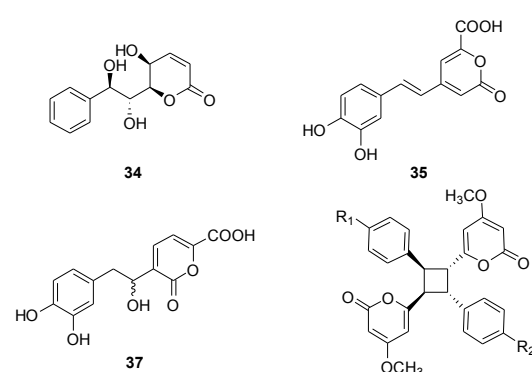

35

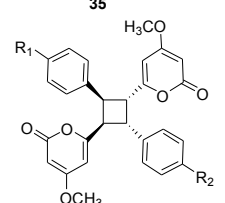

$38 \mathrm{R}_{1}=\mathrm{R}_{2}=\mathrm{OCH}_{3}$

$39 \mathrm{R}_{1}=\mathrm{OCH}_{3}, \mathrm{R}_{2}=\mathrm{H}$
$40 \mathrm{R}_{1}=\mathrm{R}_{2}=\mathrm{H}$

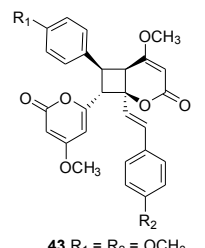

$43 \mathrm{R}_{1}=\mathrm{R}_{2}=\mathrm{OCH}_{3}$

$44 \mathrm{R}_{1}=\mathrm{OCH}_{3}, \mathrm{R}_{2}=\mathrm{H}$
$45 \mathrm{R}_{1}=\mathrm{H}, \mathrm{R}_{2}=\mathrm{OCH}$

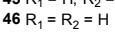

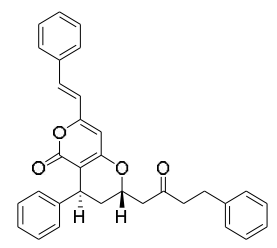

49
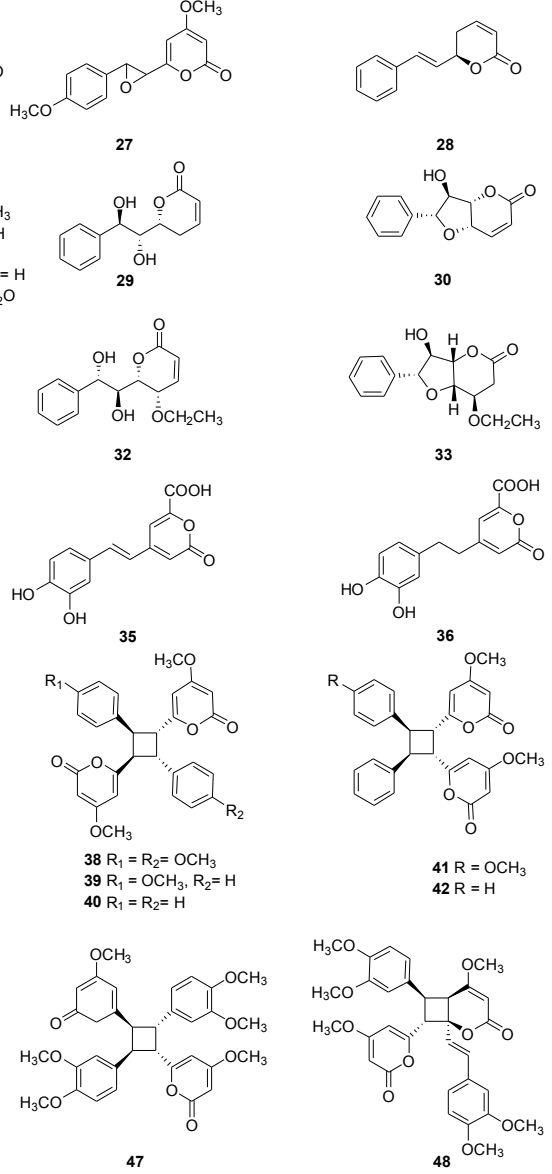

30

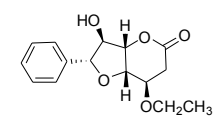

33
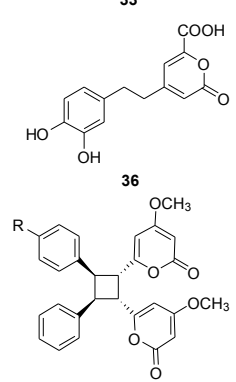

$41 \mathrm{R}=\mathrm{OCH}_{3}$ $42 \mathrm{R}=\mathrm{H}$
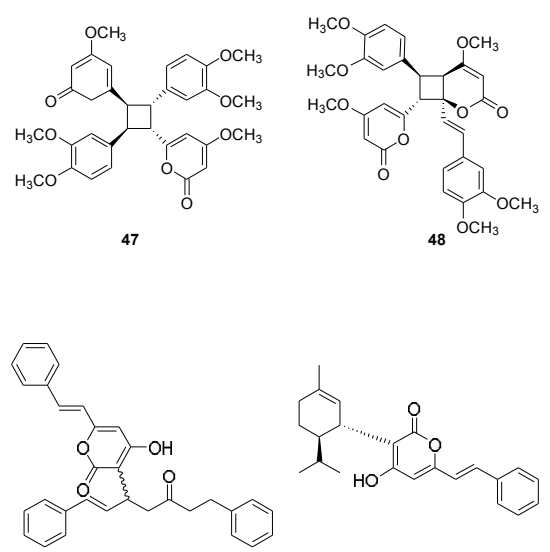

50

Figure 1 The structures of compounds 1-51.

\section{Bioactivities of kavalactones}

Among kavalactones derived from plants, the biological activities of compounds $3,7,8,18,19$ have been investigated extensively.

\section{Neuro-protective activity}

Compound 7 modulated $\gamma$-aminobutyric acid type A receptors $\left(G A B A_{A} R s\right)$ in a subtype non-selective and flumazenil-insensitive manner. ${ }^{[30]}$ Treatment of 8 activated the Nrf2 pathway and reduced neuroinflammation, hippocampal oxidative damage and memory loss in a mouse model of Alzheimer's disease. ${ }^{[31]}$ Compounds 13 and 20 exerted a protective effect against oxidative stress-induced PC12 cell death through regulation of $\mathrm{p}$-Akt and the p38 MAPK. ${ }^{[11]}$ Compounds 7,8 , and 14 can attenuate amyloid $\beta$-peptide toxicity by inducing protective gene expression mediated by Nrf2 activation in vitro. ${ }^{[32]}$

\section{Anticancer activity}

Liu et al. ${ }^{[33]}$ identified 14 as potent inducer of autophagic cell death in bladder cancer cells, and the effect of yangonin (14) inducing autophagy is associated with increased expression of beclin and ATG5. Dai et al. ${ }^{[34]}$ showed that compound 3 induced dose-dependent and time-dependent antiproliferative effects against human osteosarcoma (MG-63) cell growth. The percentage of apoptotic cells increased from $6.63 \%$ in untreated control to $23.92 \%, 23.81 \%$ and $93.9 \%$ in 25,75 , and $100 \mu \mathrm{M}$ dihydromethysticin treated cells.

\section{Hepatoprotective activity}

Compound 13 inhibited LPS-induced production of NO and iNOS expression in murine RAW 264.7 macrophages, and prevented LPS/D-GaIN-induced acute hepatitis in ICR mice. ${ }^{[10]}$

\section{Anti-osteoporosis activity}

Compounds $\mathbf{1 3}$ and 20, two promising compounds isolated from Alpinia zerumbet rhizomes, promoted differentiation of osteoblastic MC3T3-E1 cells and increased the alkaline phosphatase activity and matrix mineralization of MC3T3-E1 cells. $^{[35]}$

\section{Anti-aging activity}

Both advanced glycation endproducts and advanced lipoxidation endproducts are implicated in many age-related chronic diseases and in protein ageing. Compounds 3, 7, and 8 were identified as advanced glycation endproduct inhibitors. With $I_{50}$ values of $43.5 \pm 1.2 \mu \mathrm{M}$ and $45.0 \pm 1.3 \mu \mathrm{M}$ for 7 and $\mathbf{8}$, respectively, and the kavalactones inhibited the in vitro protein glycation significantly better than aminoguanidine $\left(\mathrm{IC}_{50}=231.0\right.$ $\pm 11.5 \mu \mathrm{M} ; p=0.01$ ), an established reference compound. ${ }^{[36]}$

\section{Hepatotoxicity}

With case reports of kava hepatotoxicity, hepatotoxicity of main constituents of kava, kavalactones, were evaluated. Hepatotoxicity of compounds 7, 8 and $\mathbf{1 4}$ on human hepatocytes (HepG2) was assessed using lactate dehydrogenase $(\mathrm{LDH})$ and ethidium bromidemide assays by Zou et al. They concluded that compound $\mathbf{3}$ had minimal cytotoxicity, compound 8 had moderate concentration-dependent toxicity, and compound 14 showed obvious toxicity with about $40 \%$ reduction in viability in the EB assay. ${ }^{[37]}$ Hepatotoxicity of compounds $\mathbf{7}$ and $\mathbf{8}$ was tested in isolated, perfused livers from rats, which were pretreated with the macrophage intoxicant gadolinium chloride. In kavalactone-perfused rat livers, hepatic sinusoids displayed extensive damage to the endothelium, but the damage could be reduced by pretreatment with gadolinum chloride. ${ }^{[38]}$

\section{Conclusions}

Kavalactones, existing in the genera Piper, Alpinia, 
Amomum, Polygala, Goniothalamus, and Dumortiera, were mostly isolated from the roots of the plants. Their various bioactivities were investigated such as neuro-protective, anti-cancer, hepatoprotective, anti-osteoporosis, and anti-aging activity. After kava ban in Europe, attention on their toxicities ${ }^{[37-40]}$ instead of effective therapeutic activities was paid on kavalactones. However, as the ban being lifted from Germany, concerns about their therapeutic value might come back again. This review provides a basis for the future research in the field of kavalatones.

\section{Acknowledgement}

This work was jointly supported by the Social Development Supportive Program of Science \& Technology Department of Guizhou Province (No.[2018]2767), the Joint Fund by Science \& Technology Bureau of Guiyang and Guizhou Medical University (No. [2017]30-37), the Joint Fund by Department of Science \& Technology of Guizhou Province and Guizhou Medical University (No. LH [2015]7339), the Fund of Innovation Team of Guizhou Province (No. 2015-4025), the Fund of Innovated Team of the Education Department of Guizhou Province (No. 2014-31).

\section{References}

[1] Kormann, E. C.; Amaral, P. A.; David, M.; Eiflerlima, V. L.; Cechinel, F. V.; Campos, B. F. Pharmacol. Rep. 2012, 64,1419.

[2] Rowe, A.; Ramzan. I. Phytother. Res. 2012, 26, 1768.

[3] Teschke, R.; Qu, S. X.; Xuan, T. D.; Lebot, V. Phytother. Res. 2011, 25, 1263.

[4] Teschke, R.; Qiu, S. X.; Lebot, V. Digest. Liver. Dis. 2011, 43, 676.

[5] Nerurkar, P. V.; Dragull, K.; Tang, C. S. Toxicol. Sci. 2004, 79, 106.

[6] Zou, L.; Harkey, M. R.; Henderson, G. L.; Dike, L. E. Planta Med. 2004, 70, 289.

[7] Kuchta, K.; Schmidt, M.; Nahrstedt, A. Planta Med. 2015, 81,1647.

[8] Bilia, A. R.; Scalise, L.; Bergonzi, M. C.; Vincieri, F. F. J. Chromatogr. B Anal. Technol. Biomed. Life Sci. 2004, 812, 203.

[9] Ganzera, M.; Khan, I. A. Chromatographia 1999, 50, 649.

[10] Chou, T. W.; Feng, J. H.; Huang, C. C.; Cheng, Y. W.; Chien, S. C.; Wang, S. Y.; Chou, T. W. PLoS One 2013,8, e77626.

[11] Rao, Y. K.; Shih, H. N.; Lee, Y. C.; Cheng, W. T.; Hung, H. C.; Wang, H. C. J. Biosci. Bioeng. 2014, 118, 679.

[12] Chate, W.; Nuntawong, N. Biochem. Syst. Ecol. 2015, 63, 34

[13] Dharmaratne, H. R.; Nanayakkara N. P.; Khan I. A. Phytochemistry 2002, 59, 429.

[14] Kuster, R. M.; Mpalantinos, M. A.; Holanda, M. C.; Lima, P.; Brand, E. T.; Parente, J. P. J. High. Resol. Chromatogr.1999, 22, 129.

[15] Pizzolatti, M. G.; Luciano, C.; Monache, F. D. Phytochemistry 2000, $55,819$.

[16] Matsuda, H.; Hirata, N.; Kawaguchi, Y.; Naruto, S.; Kubo, M. Biol. Pharm. Bull. 2006, 29, 834.
[17] Cavalheiro, A. J.; Yoshida, M. Phytochemistry 2000, 53, 811.

[18] Surivet, J. P.; Vatele, J. M. Tetrahedron 1999, 55, 13011.

[19] Bermejo, A; Léonce, S.; Cabedo, N.; Andreu, I; Caignard, D. H.; Atassi, G. J. Nat. Prod. 1999, 62, 1106.

[20] Bermejo, A.; Blázquez, M. A.; Rao, K. S.; Cortes, D. Phytochemistry 1998, 47, 1375.

[21] Bermejo, A.; Blázquez, M. A.; Serrano, A.; Z. P.; Cortes, D. J. Nat. Prod. 1997, 60, 1338.

[22] Alkofahi, A.; Ma, W. W.; Mckenzie, A. T.; Byrn, S. R.; Mclaughlin, J. L. J. Nat. Prod. 1989, 52, 1371.

[23] Kraut, L.; Mues, R.; Speicher, A.; Wagmann, M.; Eicher, T. Phytochemistry 1996, 42, 1693.

[24] Yuan, Y.; Yang, J. X.; Nie, L. H., Li, B. L.; Qin, X. B.; Wu, J. W.; Qu, S. X. J. Asian Nat. Prod. Res. 2017, DOI: 10.1080/10286020.2017. 1367768

[25] Song, J. L.; Li, B. L.; Yuan, Y.; Nie, L. H.; Niu, J.; Chiu, D.; Xu, Z. F.; Wu, J. W.; Qu, S. X. Nat. Prod. Res. 2017, 31, 2459.

[26] Rossi, M. H.; Yoshida M.; Maia, J. G. S. Phytochemistry 1997, 45, 1263.

[27] Yang, Y.; Kinoshita, K.; Koyama, K.; Takahashi, K.; Tai, T.; Nunoura, Y.; Watanabe, K. J. Nat. Prod. 1999, 12, 1672.

[28] Wang, X. B.; Yang, C. S.; Hua, S. Z.; Kong, L. Y. Chin. J. Nat. Med. 2010, 8, 419

[29] Li, Y. Y.; Chou, G. X.; Wang, Z. T. Helv. Chim. Acta 2010, 93, 382.

[30] Chua, H. C.; Christensen, E. T. H.; Hoestgaard-Jensen, K.; Hartiadi, L. Y.; Ramzan, I.; Jensen, A. A.; Absalom N.; Chebib, M. PLoS One 2016, 11, e0157700.

[31] Fragoulis, A.; Siegl, S.; Fendt, M.; Jansen, S.; Soppa, U.; Brandenburg, L. O.; Pufe, T.; Weis, J.; Wruck, C. J. Redox Biol. 2017,12, 843.

[32] Wruck, C. J.; Götz, M. E.; Herdegen, T.; Varoga, D.; Brandenburg, L. O.; Pufe, T. Mol. Pharmacol. 2008, 73, 1785.

[33] Liu, Z. B.; Ha, U.; Yu, K.; Wu, C. L.; Yokoyama, N.; Zi, X. L. J. Biomed. Res. 2017, 31, 408.

[34] Dai, J. Q.; Huang, Y. G., He, A. N. Int. J. Clin. Exp. Pathol. 2015, 8 , 4356.

[35] Kumagai, M.; Mishima, T.; Watanabe, A.; Harada, T.; Yoshida, I.; Fujita, K.; Watai, M.; Tawata, S.; Nishikawa, K.; Morimoto, Y. Biosci. Biotechnol. Biochem. 2016, 7, 1425.

[36] Upadhyay, A.; Tuenter, E.; Ahmad, R.; Amin, A.; Exarchou, V.; Apers, S.; Hermans, N. Planta Med. 2014, 80, 1001.

[37] Zou, L, Henderson, G. L.; Harkey, M. R.; Sakai, Y.; Li, A. Phytomedicine 2004, 11, 285.

[38] Zhang, L.; Rowe, A.; Braet, F.; Ramzan, I. J. Toxicol. Sci. 2012, 37, 447.

[39] Whitton, P. A.; Lau, A.; Salisbury, A.; Whitehouse, J.; Evans, C. S. Phytochemistry 2003, 64, 673

[40] Anke, J., Fu, S.; Ramzan, I. Phytomedicine 2006, 13, 192.

Received February 5, 2018

Accepted March 19, 2018 